\title{
Frozen-Density Embedding Based Many-Body Expansions
}

\author{
Daniel Schmitt-Monreal, Christoph R. Jacob ${ }^{1}$
}

Technische Universität Braunschweig, Institute of Physical and Theoretical Chemistry,

Gaußstraße 17, 38106 Braunschweig, Germany

Date: $\quad$ March 06, 2020

${ }^{1}$ E-Mail: c.jacob@tu-braunschweig.de 


\begin{abstract}
Fragmentation methods allow for the accurate quantum-chemical treatment of large molecular clusters and materials. Here, we explore the combination of two complementary approaches to the development of such fragmentation methods: the many-body expansion (MBE) on the one hand and subsystem density-functional theory (DFT) or frozen-density embedding (FDE) theory on the other hand. First, we assess potential benefits of using FDE to account of the environment in the subsystem calculation performed within the MBE. Second, we use subsystem DFT to derive a density-based MBE, in which a many-body expansion of the electron density is used to calculate the systems' total energy. This provides a correction to the energies calculated with a conventional, energy-based MBE that only depends on the subsystem's electron densities. For the test case of clusters of water and of aspirin, we show that such a density-based MBE converges faster than the conventional energy-based MBE. For our test cases, truncation errors in the interaction energies are below chemical accuracy already with a two-body expansion. The density-based MBE thus provides a promising avenue for accurate quantum-chemical calculation of molecular clusters and materials.
\end{abstract}




\section{Table of Contents Graphics}

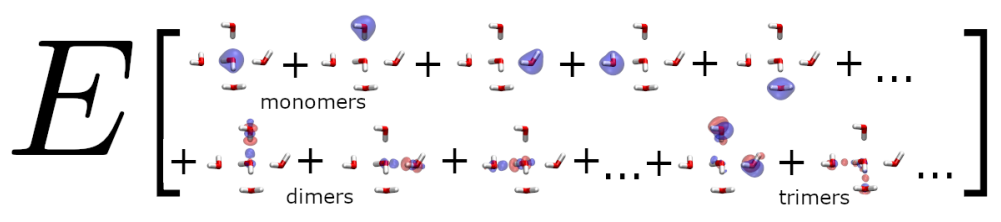

\section{Table of Contents Text}

The combination of the many-body expansion with frozen-density embedding theory is explored. A density-based many-body expansion is presented, which is able to provide interaction energies of molecular clusters with chemical accuracy already with a two-body expansion. 


\section{Introduction}

The development of fragmentation methods for quantum chemistry in the past decades enables researchers to tackle chemical systems of increasing complexity with accurate computational methods [1-4]. By partitioning a large molecular or bulk-phase system into smaller subsystems, fragmentation methods allow for its efficient, yet accurate quantumchemical treatment. On the one hand, fragmentation methods make it possible to focus on one or a few subsystems of interest (e.g., a reaction center or a chromophore), which are embedded in a complex environment $[5,6]$. Numerous embedding strategies have been developed to account for environmental effects on the subsystems of interest, ranging from continuum solvation models $[7,8]$ over classical QM/MM embedding schemes [9-11] to quantum embedding methods $[12-16]$. The latter maintain a quantum-mechanical description of the environment, which enters the quantum-chemical calculation of the subsystems of interest either via its electron density [12-14] or via its one-electron orbitals $[15,16]$. Quantum embedding methods have, for instance, been employed for the prediction of local spectroscopic properties in solution [17-19] and in biomolecular environments [20-22], for the computation of reaction energetics in enzymes [23,24], and for modeling of the quantum dynamics in scattering experiments [25].

On the other hand, fragmentation methods can be used to efficiently obtain properties of large chemical systems in which all subsystems need to be treated on an equal footing [26-29]. An important and still challenging example is the prediction of energy differences between conformers of large molecular clusters or between different polymorphs of molecular crystals [30]. Here, a balanced treatment of different types of intermolecular interactions (e.g., hydrogen bonding, $\pi-\pi$ stacking, $\mathrm{C}-\mathrm{H} \cdots \pi$ interactions, dipole-dipole interaction, dispersion interactions) is required, which demands the use of high-level quantum chemical methods [31,32]. However, such methods show a steep scaling of the computational cost with system size that precludes their direct application to larger clusters 
or bulk-phase systems. Then again, molecular clusters and crystals can be naturally partitioned into molecular subsystems and thus appear to be ideally suited for fragmentation methods.

A straightforward and conceptually simple fragmentation approach to molecular clusters and crystals is provided by the many-body expansion (MBE) [33-40], in which quantumchemical calculations for subsystems of increasing size (i.e., monomers, dimers, trimers, etc.) are used to expand the systems' total energy as,

$$
E_{\mathrm{tot}}=\sum_{I} E_{I}^{(1)}+\sum_{I<J} \Delta E_{I J}^{(2)}+\sum_{I<J<K} \Delta E_{I J K}^{(3)}+\cdots
$$

Here, $E_{I}^{(1)}=E_{I}$ is the energy of the $I$-th monomer, the interaction energy $\Delta E_{I J}^{(2)}$ of the dimer consisting of monomers $I$ and $J$ is calculated as,

$$
\Delta E_{I J}^{(2)}=E_{I J}-E_{I}-E_{J},
$$

and the trimer interaction energy is obtained as,

$$
\Delta E_{I J K}^{(3)}=E_{I J K}-\Delta E_{I J}^{(2)}-\Delta E_{I K}^{(2)}-\Delta E_{J K}^{(2)}-E_{I}-E_{J}-E_{K} .
$$

Higher-order interaction energies are defined analogously (see Refs. [37,41]). Such an MBE can be applied in combination with any quantum-chemical method and the individual quantum-chemical calculations can easily be performed in an embarrassingly parallel fashion (see, e.g., Refs. [42,43]).

While it is ensured that the MBE converges to the exact total energy of the full supermolecular system, the number of $n$-mers increases rapidly at higher orders. Therefore, in practice the MBE is only useful if it can be truncated at low order, and strategies of accelerating the convergence of the MBE are necessary if one seeks to treat larger molecular clusters or molecular crystals accurately with high-level quantum-chemical methods.

To this end, two complementary strategies are commonly pursued (see Ref. [40] for a comprehensive review). First, the MBE can be combined with embedding methods for the 
individual subsystem calculations [44-46]. By accounting for the effect of the environment on the subsystems this makes it possible to partly include higher-order many-body effects in the low-order contributions. So far, classical embedding schemes are widely used within the MBE, e.g., by using point charges extracted from monomer calculations or by using partial-charge parametrizations from suitable force fields [47]. While quantum embedding schemes have been shown to be able to account for environment effects on local molecular properties more accurately than classical embedding methods $[5,48,49]$, their use within the MBE remains mostly unexplored. Exceptions are the use of the XPol approach in the variational many-body method [50] as well as different variants of the fragment-molecular orbital method [51-53]. Recently, Head-Gordon and coworkers [54] have assessed the use of a quantum embedding scheme within the MBE by applying the embedded mean-field theory of Manby and Miller [55] for the subsystem calculations.

Second, cheap low-level computational methods can be applied to calculate the higherorder contributions that are otherwise neglected in a truncated MBE, following the idea of the ONIOM approach [56-58]. This can be used to construct multi-level composite methods based on the MBE $[59,60]$. The hybrid many-body interactions (HMBI) method of Beran and coworkers [61,62], which has been successfully applied to calculate energy differences between polymorphs of molecular crystals, uses an MBE up to twobody contributions in combination with a classical polarizable force-field to account for many-body polarization effects. While such ONIOM-style composite approaches are in principle complementary to the use of an embedded MBE, it is often unclear how to avoid double counting when combining these two strategies.

Here, we apply quantum embedding within both of these strategies for accelerating the convergence of the MBE for molecular clusters. Specifically, as quantum embedding approach we use frozen-density embedding (FDE) theory [14,63], which provides a formally exact subsystem density-functional theory (DFT) method [12]. In FDE, the embedding 
potential as well as the interaction energies between subsystems only depend on the subsystems' electron densities, which makes it possible to incorporate arbitrary quantumchemical methods into the FDE framework [64-66]. First, we explore possible advantages of using FDE as an embedding method for the subsystem calculations within the MBE. Second, we propose a density-based MBE that makes use of the framework of FDE by starting from a many-body expansion of the electron density. This provides an interaction energy correction that accurately accounts for the higher-order energy contributions that are neglected in a conventional truncated MBE.

This work is organized as follows. In Sections 2.1 and 2.2 we review the conventional, energy-based MBE as well as different embedding schemes that can be applied for the subsystem calculations. For the test case of water clusters, in Section 2.3 we then assess the convergence of the truncated MBE performed using isolated as well as embedded subsystems. In Section 2.4, the many-body convergence of the electron density is analyzed. In Section 3.1, we present the theory of our density-based MBE, which is subsequently tested and analyzed for water clusters (Sect. 3.2) and for clusters of aspirin (Sect. 3.2). Finally, a summary and conclusions are presented in Sect. 4. Details of our implementation and of the computations are given at the end of the article.

\section{Energy-Based Many-Body Expansion}

\subsection{Isolated Many-Body Expansion}

The many-body expansion decomposes the total energy of a system consisting of $N$ molecular fragments into one-body, two-body, three-body, etc. contributions [cf. Eq. (1)],

$$
E_{\mathrm{tot}}=E^{(1)}+\Delta E^{(2)}+\Delta E^{(3)}+\cdots=E^{(1)}+\sum_{i=2}^{N} \Delta E^{(i)},
$$


with the one-body contribution $E^{(1)}=\sum_{I} E_{I}^{(1)}$, the two-body contribution $\Delta E^{(2)}=$ $\sum_{I<J} \Delta E_{I J}^{(2)}$, the three-body contributions $\Delta E^{(3)}=\sum_{I<J<K} \Delta E_{I J K}^{(3)}$ and so on $[37,40]$. These $n$-body contributions are obtained from calculations for subsystems consisting of up to $n$ molecular fragments. By construction, this many-body decomposition of the total energy is exact if all terms up to order $N$ are included.

The above expression corresponds to an energy-based many-body expansion (eb-MBE), i.e., it considers only the total energies obtained from the calculation of the monomer, dimer, trimer, etc. subsystems. Conventionally, these are obtained from quantum-chemical calculations that consider the isolated subsystems (i.e., the remaining molecular fragments are completely ignored). We will refer to this type of many-body expansion as isolated energy-based many-body expansion.

Within density-functional theory (DFT) $[67,68]$, the total energy of the subsystem consisting of molecular fragments $\{I, J, \ldots\}$ is calculated as,

$$
E_{I J \ldots}\left[\rho_{I J \ldots]}\right]=T_{s}\left[\rho_{I J \ldots]}\right]+V_{\mathrm{nuc}}^{(I J \ldots)}\left[\rho_{I J \ldots}\right]+J\left[\rho_{I J \ldots}\right]+E_{\mathrm{xc}}\left[\rho_{I J \ldots}\right]+E_{\mathrm{NN}}^{(I J \ldots)},
$$

where $\rho_{I J \ldots}$ denotes the electron density of the subsystem consisting of molecular fragments $\{I, J, \ldots\}$. Here, the nuclei-electron attraction energy can be decomposed into contributions due to the nuclei of each of the molecular fragments, $V_{\text {nuc }}^{(I J \cdots)}\left[\rho_{I J \ldots}\right]=$ $\sum_{K \in\{I, J, \ldots\}} V_{\text {nuc }}^{(K)}\left[\rho_{I J \ldots}\right]$ where

$$
V_{\text {nuc }}^{(K)}\left[\rho_{I J \ldots}\right]=\int \rho_{I J \ldots}(\boldsymbol{r}) v_{\text {nuc }}^{(K)}(\boldsymbol{r}) \mathrm{d}^{3} r \quad \text { with } \quad v_{\text {nuc }}^{(K)}(\boldsymbol{r})=\sum_{i \in K} \frac{Z_{i}}{\left|\boldsymbol{r}-\boldsymbol{R}_{i}\right|} .
$$

and the nuclear repulsion energy can be decomposed into contributions of pairs of molecular fragments, $E_{\mathrm{NN}}^{(I J \ldots)}=\frac{1}{2} \sum_{K, L \in\{I, J, \ldots\}} E_{\mathrm{NN}}^{(K L)}$ with

$$
E_{\mathrm{NN}}^{(K L)}=\sum_{k \in K} \sum_{\substack{l \in L \\ l \neq k}} \frac{Z_{k} Z_{l}}{\left|\boldsymbol{R}_{k}-\boldsymbol{R}_{l}\right|}
$$

The electronic Coulomb repulsion energy is given by

$$
J\left[\rho_{I J \ldots}\right]=\frac{1}{2} \iint \frac{\rho_{I J \ldots}(\boldsymbol{r}) \rho_{I J \ldots}\left(\boldsymbol{r}^{\prime}\right)}{\left|\boldsymbol{r}-\boldsymbol{r}^{\prime}\right|} \mathrm{d}^{3} r \mathrm{~d}^{3} r^{\prime} .
$$


Finally, the noninteracting kinetic energy $T_{s}\left[\rho_{I J \ldots}\right]$ is evaluated from the $\mathrm{KS}$ orbitals $\left\{\phi_{i}\right\}$ of the KS reference system with electron density $\rho_{I J \ldots}$ and the exchange-correlation (xc) energy $E_{\mathrm{xc}}\left[\rho_{I J \ldots}\right]$ is commonly evaluated using an approximate xc functional.

In the isolated eb-MBE case, the subsystem densities $\rho_{I J \ldots}$ are obtained by minimizing the total energy functional of Eq. (5), which leads to the well-known Kohn-Sham (KS) equations for the subsystem's KS orbitals. No additional terms due to the remaining molecular fragments enter into these KS equations.

While in the following we will mostly use the conceptual framework of DFT, the subsystem energies can also be obtained with wavefunction-based quantum-chemical methods [6466]. In this case, the total energy of the isolated subsystem consisting of molecular fragments $\{I, J, \ldots\}$ is calculated as,

$$
E_{I J \ldots}=\left\langle\Psi_{I J \ldots}\left|\hat{H}^{(I J \cdots)}\right| \Psi_{I J \ldots}\right\rangle
$$

and the wavefunction $\Psi_{I J \ldots}$ is obtained as an (approximate) eigenfunction of the subsystem's Hamiltonian $\hat{H}^{(I J \cdots)}=\hat{T}+\hat{V}_{\text {nuc }}^{(I J \cdots)}+\hat{V}_{e e}+E_{\mathrm{NN}}^{(I J \cdots)}$. Again, in the case of the isolated eb-MBE, no additional terms due to the remaining molecular fragments enter into this Hamiltonian.

In the energy-based many-body expansion, $N$ quantum-chemical calculations for monomers are required to obtain the one-body contributions, $N(N-1) / 2$ calculations for dimers to obtain the two-body contributions, and $\left(\begin{array}{l}N \\ n\end{array}\right)$ calculations for $n$-mers to obtain the $n$-body contributions. A computational advantage compared to a calculation for the full system can only be exploited if the many-body expansion is truncated at a sufficiently low order $n \ll N$, i.e.,

$$
E_{\mathrm{tot}} \approx E_{\mathrm{eb}}^{(n)}=E^{(1)}+\sum_{i=2}^{n} \Delta E^{(i)} .
$$

Thus, whether the many-body expansion is useful as a tool for the quantum-chemical treatment of large molecular clusters and crystals is dependent on a fast convergence of 
the many-body expansion.

\subsection{Embedded Many-Body Expansion}

A commonly used strategy for accelerating the convergence of the eb-MBE is to approximately include the environment into the quantum-chemical calculations performed for the subsystems [44-47]. For the subsystem composed of the molecular fragments $\{I, J, \ldots\}$,

the effect of this environment is included as a (local) embedding potential, $v_{\mathrm{emb}}^{(I J \cdots)}(\boldsymbol{r})$, which is due to the remaining molecular fragments $K \notin\{I, J, \ldots\}$.

Within the framework of DFT, this embedding potential enters the KS equations for the considered subsystem, whereas within wavefunction-based calculations it enters via an embedding operator $\hat{V}_{\mathrm{emb}}^{\{I J \cdots\}}=\sum_{i} v_{\mathrm{emb}}^{(I J \cdots)}\left(\boldsymbol{r}_{i}\right)$. Thus, it will change the subsystem's electron density $\rho_{I J \ldots}$ or its wavefunction $\Psi_{I J \ldots}$, which is subsequently used to evaluate the subsystem's energy according to Eq. (5) or Eq. (9), respectively. Note that no explicit embedding energy contributions enter the eb-MBE, i.e., there are no contributions of the embedding potential in Eqs. (5) and (9). Consequently, only the energy terms that refer to the embedded subsystems are included in the expansion of Eq. (1). This is necessary to prevent double counting of such contributions in the eb-MBE. Note that for technical reasons in some implementations, embedding energy contributions are included in the subsystem energies, but are subsequently removed via a suitable self-energy correction $[69,70]$.

A widely used example for such an embedded eb-MBE is the electrostatically embedded many-body expansion (EE-MBE) $[44,45]$, which uses the embedding potential,

$$
v_{\mathrm{emb}, \mathrm{PC}}^{(I J \ldots)}(\boldsymbol{r})=\sum_{K \notin\{I, J, \ldots\}} \sum_{k \in K} \frac{q_{k}}{\left|\boldsymbol{r}-\boldsymbol{R}_{k}\right|}
$$

where $q_{k}$ are suitable partial charges placed at the positions of the nuclei $\boldsymbol{R}_{k}$. Such an electrostatic point-charge embedding is commonly used in the context of QM/MM 
methods. It has been previously shown that it can accelerate the convergence of the eb-MBE for molecular clusters compared to the isolated eb-MBE $[44,45,71,72]$.

As an alternative to an electrostatic point-charge embedding, we also consider the embedding potential of frozen-density embedding (FDE) theory [12,14,63],

$$
\begin{aligned}
v_{\mathrm{emb}, \mathrm{FDE}}^{(I J \cdots)}(\boldsymbol{r})= & \sum_{K \notin\{I, J, \ldots\}} v_{\mathrm{nuc}}^{(K)}(\boldsymbol{r})+\sum_{K \notin\{I, J, \ldots\}} \int \frac{\rho_{K}\left(\boldsymbol{r}^{\prime}\right)}{\left|\boldsymbol{r}-\boldsymbol{r}^{\prime}\right|} \mathrm{d}^{3} r^{\prime} \\
& +\left.\frac{\delta T_{s}[\rho]}{\delta \rho}\right|_{\rho=\rho_{\mathrm{tot}}}-\left.\frac{\delta T_{s}[\rho]}{\delta \rho}\right|_{\rho=\rho_{I J} \ldots}+\left.\frac{\delta E_{\mathrm{xc}}[\rho]}{\delta \rho}\right|_{\rho=\rho_{\mathrm{tot}}}-\left.\frac{\delta E_{\mathrm{xc}}[\rho]}{\delta \rho}\right|_{\rho=\rho_{I J} \ldots}
\end{aligned}
$$

with $\rho_{\text {tot }}=\rho_{I J \ldots}+\sum_{K \notin\{I, J, \ldots\}} \rho_{K}$. This embedding potential contains the full electrostatic interaction as well as non-classical contributions due to the exchange-correlation and kinetic energy. It is formally exact in the sense that with the exact functionals and suitable environment electron densities, it will lead to the exact total density [73, 74]. However, in practice an approximate local xc functional as well as an approximate nonadditive kinetic-energy functional have to be used $[12,75,76]$.

Compared to an electrostatic point-charge embedding, the FDE embedding potential does not rely on a parametrization of point charges. Furthermore, by fully accounting for the electrostatic as well as non-classical interactions it should be able to describe the effect of the environment on the considered active subsystem more accurately. It has previously been shown that for the calculation of spectroscopic properties, FDE is usually superior to a simple point-charge embedding as used in conventional QM/MM methods $[5,48,49,66]$. Here, we want to explore possible advantages of the FDE embedding potential within the eb-MBE. The different variants of the MBE that are studied in this work are summarized in Table I. 
Table I: Overview of the different variants of the many-body expansion (MBE) and of embedding schemes that are studied in this work, along with the corresponding acronyms used in the figures and tables.

\begin{tabular}{lll}
\hline \hline MBE variant & acronym & definition \\
\hline energy-based & eb-MBE & Eq. (10) \\
density-based & db-MBE & Eq. (22) \\
\hline \hline embedding scheme & acronym & $v_{\text {emb }}^{(I J \cdots)}(\boldsymbol{r})$ \\
\hline isolated & iso & - \\
point charges & PC & Eq. (11) \\
frozen-density & FDE & Eq. (12) \\
\hline \hline
\end{tabular}

\subsection{Test Case: Water Clusters}

Water clusters present a challenging test case for the MBE because of the large many-body polarization effects that need to be captured [38,44,72,77-80]. Here, we consider water clusters extracted from the crystal structure of ice $\mathrm{I}_{\mathrm{h}}[81,82]$. First, we use a water pentamer, $\left(\mathrm{H}_{2} \mathrm{O}\right)_{5}$, in which the central water molecule is surrounded by four water molecules in a pseudo-tetrahedral environment (see Fig. 1a). Second, we consider an $\left(\mathrm{H}_{2} \mathrm{O}\right)_{17}$ cluster, in which a second shell of water molecules has been added to the pentamer, i.e., each of the surrounding water molecules in the pentamer is now coordinated to three additional water molecules that complete its pseudo-tetrahedral environment (see Fig. 1b).

We aim at calculating the interaction energy per molecule between the water molecules in these clusters, i.e.,

$$
E_{\mathrm{int}}=\frac{1}{N}\left(E_{\mathrm{tot}}-\sum_{I} E_{I}^{(1)}\right)=\frac{1}{N}\left(E_{\mathrm{tot}}-E_{\mathrm{eb}, \text { iso }}^{(1)}\right) .
$$

Here, we normalize the interaction energy to one water molecule to allow for a better comparison between the clusters of different size. The interaction energy obtained from a supermolecular calculation of $E_{\text {tot }}$ can be used as reference, and is compared to the 
(a)
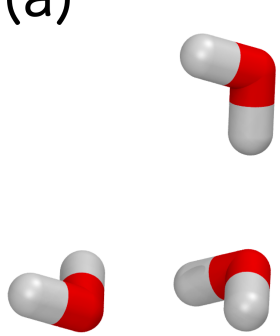
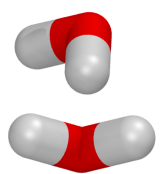

(b)

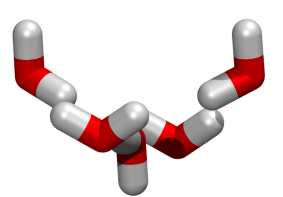

diter

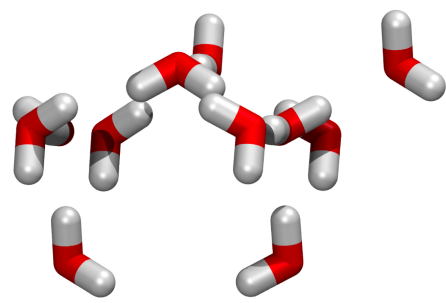

Figure 1: Structure of the water clusters considered as a test case (a) $\left(\mathrm{H}_{2} \mathrm{O}\right)_{5}$ and (b) $\left(\mathrm{H}_{2} \mathrm{O}\right)_{17}$. The structures of these clusters have been extracted from the crystal structure of ice $\mathrm{I}_{\mathrm{h}}$.

interaction energy per molecule from eb-MBEs of different orders, i.e.,

$$
E_{\text {int,eb-MBE }}^{(n)}=\frac{1}{N}\left(E_{\mathrm{eb}-\mathrm{MBE}}^{(n)}-E_{\mathrm{eb}, \mathrm{iso}}^{(1)}\right),
$$

with the truncation error per molecule of the MBE,

$$
\Delta_{\mathrm{eb}-\mathrm{MBE}}^{(n)}=\frac{1}{N}\left(E_{\mathrm{eb}-\mathrm{MBE}}^{(n)}-E_{\text {supermol }}\right) .
$$

All calculations have been performed using DFT with the BP86 xc-functional $[83,84]$ and a triple-zeta plus polarization (TZP) basis set of Slater-type orbitals [85] (see Section "Computational Details" for further information).

Table II collects the interaction energies per molecule as well as the corresponding truncation errors (compared to the supermolecular reference calculation) at different orders of eb-MBEs for $\left(\mathrm{H}_{2} \mathrm{O}\right)_{5}$ and $\left(\mathrm{H}_{2} \mathrm{O}\right)_{17}$. In addition to the isolated eb-MBE, two different embedded eb-MBEs are considered. First, a conventional electrostatic point-charge embedding is used, in which TIP3P [86] point charges $\left(q_{\mathrm{H}}=+0.417\right.$ and $\left.q_{\mathrm{O}}=-0.834\right)$ are assigned to the environment water molecules. Second, frozen-density embedding is employed. Here, we use the densities calculated for the isolated monomer subsystems to approximate the electron densities of the environment subsystems $\rho_{K}$ in Eq. (12). Note that an iterative update of all subsystem densities in freeze-and-thaw iterations $[26,87]$ 
Table II: Interaction energies per molecular fragment (in $\mathrm{kJ} / \mathrm{mol}$ ) of the water clusters $\left(\mathrm{H}_{2} \mathrm{O}\right)_{5}$ and $\left(\mathrm{H}_{2} \mathrm{O}\right)_{17}$ obtained from energy-based many-body expansions compared to the supermolecular reference value for DFT/BP86/TZP calculations. Included are the isolated eb-MBE ("iso") as well as embedded eb-MBEs using point charge embedding ("PC") as well as frozen-density embedding ("FDE"). See text for details.

\begin{tabular}{|c|c|c|c|c|c|c|c|}
\hline & \multirow[b]{2}{*}{ order } & \multicolumn{2}{|l|}{ iso } & \multicolumn{2}{|c|}{$\mathrm{PC}$} & \multicolumn{2}{|c|}{ FDE } \\
\hline & & $E_{\text {int,eb-MBE }}^{(n)}$ & $\Delta^{(n)}$ & $E_{\text {int,eb-MBE }}^{(n)}$ & $\Delta^{(n)}$ & $E_{\text {int,eb-MBE }}^{(n)}$ & $\Delta^{(n)}$ \\
\hline \multirow[t]{4}{*}{$\left(\mathrm{H}_{2} \mathrm{O}\right)_{5}$} & 2-body & -9.45 & +1.40 & -10.16 & +0.69 & -9.97 & +0.88 \\
\hline & 3-body & -10.93 & -0.08 & -10.89 & -0.04 & -10.92 & -0.07 \\
\hline & 4-body & -10.86 & -0.01 & -10.86 & +0.01 & -10.85 & 0.00 \\
\hline & supermol. & -10.85 & & -10.85 & & -10.85 & \\
\hline \multirow[t]{4}{*}{$\left(\mathrm{H}_{2} \mathrm{O}\right)_{17}$} & 2-body & -11.12 & +2.37 & -11.95 & +1.54 & -11.79 & +1.70 \\
\hline & 3-body & -13.66 & -0.17 & -13.66 & -0.17 & -13.71 & -0.22 \\
\hline & 4-body & -13.63 & -0.14 & -13.60 & -0.11 & -13.36 & +0.13 \\
\hline & supermol. & -13.49 & & -13.49 & & -13.49 & \\
\hline
\end{tabular}
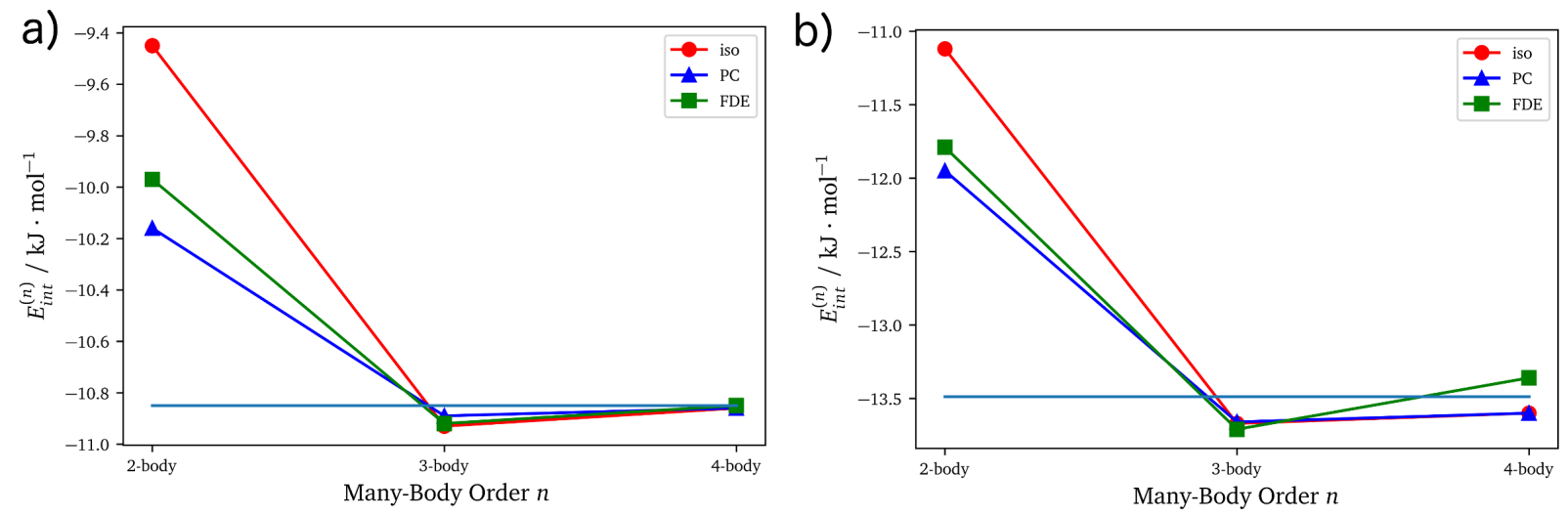

Figure 2: Convergence of the energy-based many-body expansion for the interaction energies per molecular fragment, $E_{\text {int,eb-MBE }}^{(n)}($ in $\mathrm{kJ} / \mathrm{mol})$ of $(\mathrm{a})\left(\mathrm{H}_{2} \mathrm{O}\right)_{5}$ and $(\mathrm{b})\left(\mathrm{H}_{2} \mathrm{O}\right)_{17}$ for DFT/BP86/TZP calculations. Included are the isolated eb-MBE ("iso", red circles) as well as embedded eb-MBEs using point charge embedding ("PC", blue triangles) as well as frozen-density embedding ("FDE", green squares). The supermolecular reference value are included as horizontal line. See text for details. 
would also be possible, but is not performed here. The results for the different eb-MBEs are also visualized in Fig. 2.

For the water pentamer, all eb-MBEs show the expected convergence towards the supermolecular result with increasing order of the MBE. In all cases, the supermolecular result is already reproduced with a 4-body expansion, and the 4-body contribution is below $0.1 \mathrm{~kJ} / \mathrm{mol}$ per molecule. The truncation error of the two-body expansion (i.e., the contribution of 3-body and higher-order terms) amounts to $1.40 \mathrm{~kJ} / \mathrm{mol}$ per molecule for the isolated eb-MBE. This truncation error is reduced to $0.69 \mathrm{~kJ} / \mathrm{mol}$ and $0.88 \mathrm{~kJ} / \mathrm{mol}$ per molecule for the embedded eb-MBEs with point charge embedding and frozen-density embedding, respectively. Similarly, the embedded MBEs also slightly reduce the truncation error of the 3-body expansion (i.e., the contribution of 4-body and higher-order terms).

A similar picture is obtained for $\left(\mathrm{H}_{2} \mathrm{O}\right)_{17}$, even though the truncation error per molecule are now larger than for the pentamer. With the isolated eb-MBE, the two-body truncation error amounts to $2.36 \mathrm{~kJ} / \mathrm{mol}$ per molecule, which is reduced to $1.54 \mathrm{~kJ} / \mathrm{mol}$ and $1.70 \mathrm{~kJ} / \mathrm{mol}$ per molecule for the embedded eb-MBE with point charge and frozendensity embedding, respectively. All eb-MBEs show a 3-body truncation error between -0.17 and $-0.22 \mathrm{~kJ} / \mathrm{mol}$ per molecule and a 4-body truncation error between -0.11 and $+0.13 \mathrm{~kJ} / \mathrm{mol}$ per molecule.

Altogether, our tests for two water clusters confirm that (a) 3-body (and possibly also 4body) contribution are essential for describing many-body polarization effects and cannot be neglected in the eb-MBE $[38,72]$ and (b) the truncation error (at least for the two-body expansion) of the eb-MBE can be reduced using a suitable embedding scheme $[71,72]$. However, for the embedded eb-MBEs, the two-body truncation error remains sizable and 3-body contributions thus cannot be neglected, i.e., overall the $n$-body convergence of the eb-MBE is not accelerated. This holds both for PC embedding and for FDE. This is in 
line with the previous finding that for water clusters, the embedded eb-MBE is rather insensitive to the choice of the embedding charges $[40,72,88]$.

\subsection{Many-body expansion of the electron density}

In the embedded MBE, the embedding potential accounts for the polarization of the considered subsystems due to their environment. By inspecting the electron density, it becomes possible to directly judge the accuracy of the considered embedding schemes. Therefore, we consider the many-body expansion of the total electron density as [cf. Eqs. (1) and (4)]

$$
\begin{aligned}
\rho_{\mathrm{tot}}(\boldsymbol{r}) & =\sum_{I} \rho_{I}^{(1)}(\boldsymbol{r})+\sum_{I<J} \Delta \rho_{I J}^{(2)}(\boldsymbol{r})+\sum_{I<J<K} \Delta \rho_{I J K}^{(3)}(\boldsymbol{r})+\cdots \\
& =\rho_{\mathrm{tot}}^{(1)}(\boldsymbol{r})+\Delta \rho_{\mathrm{tot}}^{(2)}(\boldsymbol{r})+\Delta \rho_{\mathrm{tot}}^{(3)}(\boldsymbol{r})+\cdots=\rho_{\mathrm{tot}}^{(1)}(\boldsymbol{r})+\sum_{i=2}^{N} \Delta \rho_{\mathrm{tot}}^{(i)}(\boldsymbol{r}),
\end{aligned}
$$

where all terms are defined in analogy to the MBE of the total energy. For instance, the two-body density corrections are defined as $\Delta \rho_{I J}^{(2)}=\rho_{I J}-\rho_{I}^{(1)}-\rho_{J}^{(1)}$ and the 3-body density corrections are given by $\Delta \rho_{I J K}^{(3)}=\rho_{I J K}-\Delta \rho_{I J}^{(2)}-\Delta \rho_{I K}^{(2)}-\Delta \rho_{J K}^{(2)}-\rho_{I}^{(1)}-\rho_{J}^{(1)}-\rho_{K}^{(1)}$. As for the the total energy, the MBE can be performed using calculations for isolated or embedded subsystems, and the total electron density can be approximated by a truncated MBE,

$$
\rho_{\mathrm{tot}}(\boldsymbol{r}) \approx \rho_{\mathrm{tot}}^{(n)}(\boldsymbol{r})=\rho_{\mathrm{tot}}^{(1)}(\boldsymbol{r})+\sum_{i=2}^{n} \Delta \rho_{\mathrm{tot}}^{(i)}(\boldsymbol{r}) .
$$

To assess the convergence of the isolated and embedded MBEs for the electron density, Fig. 3 shows isosurface plots of the difference densities of the many-body expanded electron densities compared to the one obtained from a supermolecular calculation,

$$
\Delta \rho_{\mathrm{MBE}}^{(n)}(\boldsymbol{r})=\rho_{\mathrm{tot}}(\boldsymbol{r})-\rho_{\mathrm{tot}}^{(n)}(\boldsymbol{r})
$$



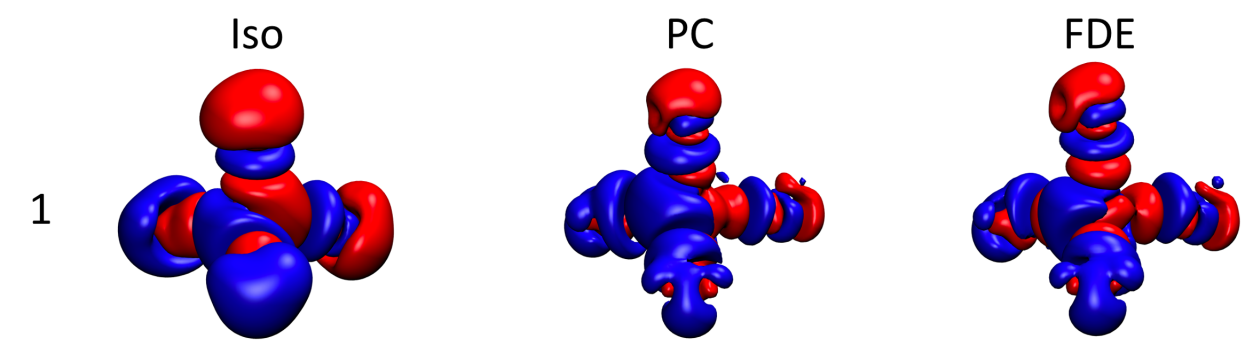

2
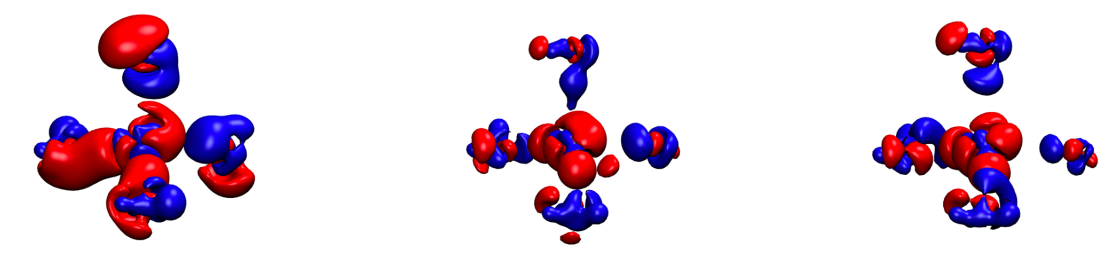

3
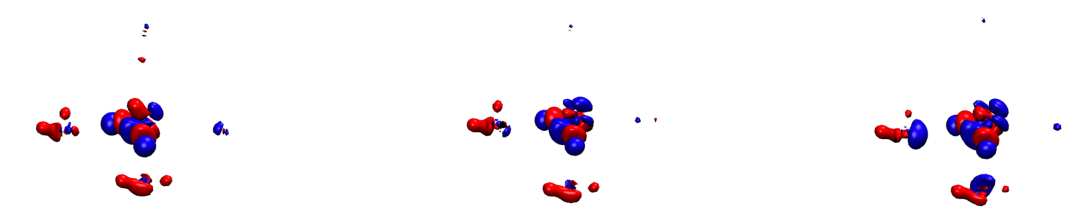

Figure 3: Isosurface plots (isovalue $2.0 \cdot 10^{-4} \mathrm{e} / \mathrm{bohr}^{3}$ for one-body and $0.7 \cdot 10^{-4} \mathrm{e} / \mathrm{bohr}^{3}$ for 2-body and 3-body expansion) of the truncation error in the total electron density $\Delta \rho_{\mathrm{MBE}}^{(n)}(\boldsymbol{r})$ [see Eq. (18)] at different orders $n$ of the isolated MBE ("iso") and of embedded MBEs using point-charge embedding ("PC") and frozen-density embedding ("FDE") for $\left(\mathrm{H}_{2} \mathrm{O}\right)_{5}$. With a 4-body expansion, the difference densities are not visible anymore with the chosen isovalue.

for the test case of the water pentamer. For a quantitative assessment, the integrated density errors,

$$
\left|\Delta \rho_{\mathrm{MBE}}^{(n)}\right|=\int\left|\rho_{\mathrm{tot}}(\boldsymbol{r})-\rho_{\mathrm{tot}}^{(n)}(\boldsymbol{r})\right| \mathrm{d}^{3} r
$$

are listed in Tab. III for the different MBEs of the electron density in both the water pentamer and $\left(\mathrm{H}_{2} \mathrm{O}\right)_{5}$.

Inspection of Fig. 3 shows that for $\left(\mathrm{H}_{2} \mathrm{O}\right)_{5}$, the convergence of the total density is significantly accelerated with the embedded MBEs compared to the isolated MBEs. Already at first order (i.e., for the sum of the fragment densities) the inclusion of an embedding potential significantly improves the electron density. Similarly, for a two-body expansion the 
Table III: Integrated density errors in the total electron density $\left|\Delta \rho_{\mathrm{MBE}}^{(n)}\right|[$ see Eq. (19)] at different orders $n$ of the isolated MBE ("iso") and of embedded MBEs using point-charge embedding ("PC") and frozen-density embedding ("FDE") for $\left(\mathrm{H}_{2} \mathrm{O}\right)_{5}$ and $\left(\mathrm{H}_{2} \mathrm{O}\right)_{17}$. All values in $\mathrm{e} / \mathrm{bohr}^{3}$.

\begin{tabular}{ccccccccc}
\hline \hline & \multicolumn{3}{c}{$\left(\mathrm{H}_{2} \mathrm{O}\right)_{5}$} & & \multicolumn{3}{c}{$\left(\mathrm{H}_{2} \mathrm{O}\right)_{17}$} \\
\cline { 2 - 3 } \cline { 7 - 9 } & iso & PC & FDE & & iso & PC & FDE \\
\hline 1-body & 0.455 & 0.196 & 0.183 & & 1.759 & 0.774 & 0.713 \\
2-body & 0.040 & 0.020 & 0.020 & & 0.194 & 0.094 & 0.098 \\
3-body & 0.006 & 0.005 & 0.006 & & 0.075 & 0.065 & 0.065 \\
4-body & 0.001 & 0.001 & 0.001 & & 0.071 & 0.069 & 0.068 \\
\hline \hline
\end{tabular}

difference densities obtained from the embedded MBEs are considerably smaller than for the isolated MBE. For a 3-body and 4-body expansion, the difference densities approach zero for both the isolated and the embedded MBEs. These observations are confirmed by the integrated density errors listed in Tab. III. At first and second order, the density error of the embedded MBEs is less than half of the one obtained in the isolated MBE, while for the 3-body and 4-body expansions very small errors of similar size are obtained in all cases. Similar observations can be made for $\left(\mathrm{H}_{2} \mathrm{O}\right)_{17}$. At first and second order, the embedded MBEs reduce the density error by more than half. For the 3-body and 4-body expansions, the embedded MBEs still improve the electron densities compared to the isolated MBE, but to a smaller extent. Note that for $\left(\mathrm{H}_{2} \mathrm{O}\right)_{17}$, the density errors in the 4-body expansion is still larger then for $\left(\mathrm{H}_{2} \mathrm{O}\right)_{5}$, which is explained by the larger number of nearest-neighbor interactions (i.e., in the 17-mer the five central water molecules are each coordinated to four other water molecules).

Thus, with embedded MBEs the electron density can already be considered converged with a two-body expansion and 3-body and higher-order contributions becomes negligible for the electron density $\left(\left|\Delta \rho_{\mathrm{MBE}}^{(n)}\right|<0.1\right)$ if an embedded MBE is used. This is in stark contrast to the slower convergence that was observed for the interaction energies. Finally, we note that as for the interaction energies, the use of a FDE embedding potential does not 
significantly improve upon the use of TIP3P point-charge embedding for the considered water clusters.

\section{Density-Based Many-Body Expansion}

\subsection{Theory}

The observation that an embedded many-body expansion converges significantly faster for the electron density than for the energy led us to the idea to exploit this faster convergence by performing a density-based MBE of the total energy as,

$$
E_{\mathrm{tot}}=E_{\mathrm{tot}}\left[\sum_{I} \rho_{I}^{(1)}(\boldsymbol{r})+\sum_{I<J} \Delta \rho_{I J}^{(2)}(\boldsymbol{r})+\sum_{I<J<K} \Delta \rho_{I J K}^{(3)}(\boldsymbol{r})+\cdots\right]
$$

that is, to use the many-body expanded density in the DFT total energy expression [cf. Eq. (5)]. By truncating the MBE of the total electron density [cf. Eq. (17)], one obtains a truncated density-based MBE (db-MBE) that can be used to approximate the total energy,

$$
E_{\mathrm{tot}} \approx E_{d b}^{(n)}=E_{\mathrm{tot}}\left[\rho_{\mathrm{tot}}^{(n)}(\boldsymbol{r})\right]
$$

Given the results of the previous section, it can be expected that this db-MBE converges faster with respect to the many-body expansion order than the conventional eb-MBE. As will be shown below, the total energy of a truncated db-MBE can be expressed as the total energy of the corresponding eb-MBE plus an interaction energy correction,

$$
E_{d b}^{(n)}=E_{\text {tot }}\left[\rho_{\text {tot }}^{(n)}(\boldsymbol{r})\right]=E_{e b}^{(n)}+E_{\text {corr }}^{(n)}
$$

When including only the first-order contribution to the total density (i.e., the total electron density is approximated as a sum of fragment densities), Eq. (20) leads to the one-body 
energy in the db-MBE,

$$
\begin{aligned}
E_{\mathrm{db}}^{(1)}=E_{\mathrm{tot}}\left[\sum_{I} \rho_{I}^{(1)}\right]= & \sum_{I} T_{s}\left[\rho_{I}^{(1)}\right]+\sum_{I} \int \rho_{I}^{(1)}(\boldsymbol{r}) v_{\mathrm{nuc}}^{(I)}(\boldsymbol{r}) \mathrm{d}^{3} r+\sum_{I} J\left[\rho_{I}^{(1)}\right] \\
& +\sum_{I} E_{\mathrm{xc}}\left[\rho_{I}^{(1)}\right]+\sum_{I} E_{\mathrm{NN}}^{(I I)}+\sum_{I \neq J} \int \rho_{I}^{(1)}(\boldsymbol{r}) v_{\mathrm{nuc}}^{(J)}(\boldsymbol{r}) \mathrm{d}^{3} r \\
& +\sum_{I<J} \iint \frac{\rho_{I}^{(1)}(\boldsymbol{r}) \rho_{J}^{(1)}\left(\boldsymbol{r}^{\prime}\right)}{\left|\boldsymbol{r}-\boldsymbol{r}^{\prime}\right|} \mathrm{d}^{3} r \mathrm{~d}^{3} r^{\prime}+\sum_{I<J} E_{\mathrm{NN}}^{(I J)} \\
& +T_{s}^{\mathrm{nadd}}\left[\left\{\rho_{I}^{(1)}\right\}\right]+E_{\mathrm{xc}}^{\mathrm{nadd}}\left[\left\{\rho_{I}^{(1)}\right\}\right]
\end{aligned}
$$

where the nonadditive kinetic and xc energy functionals are defined as

$$
\begin{aligned}
& T_{s}^{\text {nadd }}\left[\left\{\rho_{I}^{(1)}\right\}\right]=T_{s}\left[\sum_{I} \rho_{I}^{(1)}\right]-\sum_{I} T_{s}\left[\rho_{I}^{(1)}\right] \\
& E_{\mathrm{xc}}^{\mathrm{nadd}}\left[\left\{\rho_{I}^{(1)}\right\}\right]=E_{\mathrm{xc}}\left[\sum_{I} \rho_{I}^{(1)}\right]-\sum_{I} E_{\mathrm{xc}}\left[\rho_{I}^{(1)}\right] .
\end{aligned}
$$

This one-body energy can be decomposed as

$$
E_{\mathrm{db}}^{(1)}=\sum_{I} E_{I}+E_{\mathrm{corr}}^{(1)}=E_{\mathrm{eb}}^{(1)}+E_{\mathrm{corr}}^{(1)}
$$

with the one-body energy in the eb-MBE,

$$
E_{\mathrm{eb}}^{(1)}=\sum_{I} E_{I}\left[\rho_{I}^{(1)}\right]
$$

and an interaction energy correction

$$
\begin{aligned}
E_{\text {corr }}^{(1)}= & \sum_{I \neq J} \int \rho_{I}^{(1)}(\boldsymbol{r}) v_{\text {nuc }}^{(J)}(\boldsymbol{r}) \mathrm{d}^{3} r+\sum_{I<J} \iint \frac{\rho_{I}^{(1)}(\boldsymbol{r}) \rho_{J}^{(1)}\left(\boldsymbol{r}^{\prime}\right)}{\left|\boldsymbol{r}-\boldsymbol{r}^{\prime}\right|} \mathrm{d}^{3} r \mathrm{~d}^{3} r^{\prime}+\sum_{I<J} E_{\mathrm{NN}}^{(I J)} \\
& +E_{\mathrm{xc}}^{\text {nadd }}\left[\left\{\rho_{I}^{(1)}\right\}\right]+T_{s}^{\text {nadd }}\left[\left\{\rho_{I}^{(1)}\right\}\right]
\end{aligned}
$$

where the nonadditive kinetic and xc energy contributions need to be evaluated using an approximate functional that only depend on the local electron density (e.g., GGA-type functionals $[75,76])$, as is common in subsystem DFT [12]. If the electron densities $\rho_{I}^{(1)}$ are obtained from calculations for the isolated fragments, the above energy expressions is equivalent to the Harris functional $[89,90]$, whereas for electron densities obtained in 
the presence of an potential they correspond to those used in FDE theory and subsystem DFT $[12,63,91]$. Note, however, that the electron densities are not determined selfconsistently and thus do not minimize the energy functional of Eq. (23).

Even though we used the formalism of DFT to derive the above energy expressions, the above interaction energy correction $E_{\text {corr }}^{(1)}$ can be combined with energies $E_{\mathrm{eb}}^{(1)}$ obtained from the MBE with any quantum-chemical method. The evaluation of the interaction energy correction according to Eq. (28) does only require the fragment electron densities, which are also available from both DFT and wavefunction-based quantum-chemical methods.

When going beyond first order in the db-MBE, we still want to express the total energy as the sum of the energy of the truncated eb-MBE plus an interaction energy correction $E_{\text {corr }}^{(n)}$ [cf. Eq. (22)]. To obtain an expression for $E_{\text {corr }}^{(n)}$, we first realize that within the DFT formalism, the total energy is the sum of five energy contributions and an eb-MBE can be performed for each of these terms separately. Thus, in a truncated $n$-body expansion, each of these terms $\left(X=T_{s}, V_{\text {nuc }}, J, E_{x c}, E_{N N}\right)$ is approximated as,

$$
X^{(n)}=X^{(1)}+\sum_{i=2}^{n} \Delta X^{(n)} .
$$

Thus, the total energy in the truncated eb-MBE can be expressed as

$$
\begin{aligned}
E_{\mathrm{eb}}^{(n)} & =T_{s}^{(n)}+V_{\mathrm{nuc}}^{(n)}+J^{(n)}+E_{x c}^{(n)}+E_{N N}^{(n)} \\
& =E^{(1)}+\sum_{i=1}^{n}\left(\Delta T_{s}^{(n)}+\Delta V_{\mathrm{nuc}}^{(n)}+\Delta J^{(n)}+\Delta E_{x c}^{(n)}\right)+\Delta E_{\mathrm{NN}}^{(2)}
\end{aligned}
$$

Note that for the nuclear-nuclear repulsion energy, which is independent of the electron density, there are only up to two-body contributions.

By rearranging Eq. (21) and using the eb-MBE of the different energy contributions [see 
Eq. (29)], we find that for $n>1$ the $n$-body interaction energy correction is given by

$$
\begin{aligned}
E_{\mathrm{corr}}^{(n)}= & E_{\mathrm{tot}}\left[\rho_{\mathrm{tot}}^{(n)}(\boldsymbol{r})\right]-E_{\mathrm{eb}}^{(n)} \\
=( & \left.V_{\text {nuc }}\left[\rho_{\mathrm{tot}}^{(n)}\right]-V_{\mathrm{nuc}}^{(n)}\right)+\left(J\left[\rho_{\mathrm{tot}}^{(n)}\right]-J^{(n)}\right) \\
& \quad+T_{s}^{\text {nadd },(n)}\left[\left\{\rho_{I}\right\},\left\{\rho_{I J}\right\}, \ldots\right]+E_{\mathrm{xc}}^{\mathrm{nadd},(n)}\left[\left\{\rho_{I}\right\},\left\{\rho_{I J}\right\}, \ldots\right]
\end{aligned}
$$

Here, the $n$-body nonadditive kinetic and xc energy functionals are defined as

$$
\begin{aligned}
& T_{s}^{\mathrm{nadd},(n)}\left[\left\{\rho_{I}\right\},\left\{\rho_{I J}\right\}, \ldots\right]=T_{s}\left[\rho_{\mathrm{tot}}^{(n)}\right]-T_{s}^{(n)} \\
& E_{\mathrm{xc}}^{\mathrm{nadd},(n)}\left[\left\{\rho_{I}\right\},\left\{\rho_{I J}\right\}, \ldots\right]=E_{\mathrm{xc}}\left[\rho_{\mathrm{tot}}^{(n)}\right]-E_{\mathrm{xc}}^{(n)} .
\end{aligned}
$$

Again, these contributions need to be evaluated using suitable approximate density functionals $[12,75,76]$.

The electron-nuclei attraction energy functional $V_{\text {nuc }}\left[\rho_{\text {tot }}^{(n)}\right]$ and the Coulomb repulsion energy functional $J\left[\rho_{\text {tot }}^{(n)}\right]$ can be evaluated recursively for the many-body expanded electron density. For the electron-nuclei attraction energy we find,

$$
V_{\text {nuc }}\left[\rho_{\text {tot }}^{(n)}\right]=V_{\text {nuc }}\left[\rho_{\text {tot }}^{(n-1)}+\Delta \rho_{\text {tot }}^{(n)}\right]=V_{\text {nuc }}\left[\rho_{\text {tot }}^{(n-1)}\right]+\int \Delta \rho_{\text {tot }}^{(n)} v_{\text {nuc }}(\boldsymbol{r}) \mathrm{d}^{3} r
$$

and for the Coulomb energy we have,

$$
\begin{aligned}
J\left[\rho_{\mathrm{tot}}^{(n)}\right] & =J\left[\rho_{\mathrm{tot}}^{(n-1)}+\Delta \rho_{\mathrm{tot}}^{(n)}\right] \\
& =J\left[\rho_{\mathrm{tot}}^{(n-1)}\right]+\iint \frac{\rho_{\mathrm{tot}}^{(n-1)}(\boldsymbol{r}) \Delta \rho_{\mathrm{tot}}^{(n)}\left(\boldsymbol{r}^{\prime}\right)}{\left|\boldsymbol{r}-\boldsymbol{r}^{\prime}\right|} \mathrm{d}^{3} r \mathrm{~d}^{3} r^{\prime}+\frac{1}{2} \iint \frac{\Delta \rho_{\mathrm{tot}}^{(n)}(\boldsymbol{r}) \Delta \rho_{\mathrm{tot}}^{(n)}\left(\boldsymbol{r}^{\prime}\right)}{\left|\boldsymbol{r}-\boldsymbol{r}^{\prime}\right|} \mathrm{d}^{3} r \mathrm{~d}^{3} r^{\prime} .
\end{aligned}
$$

Thus, for $n>1$ the calculation of these corrections requires only the knowledge of the total electron density at expansion order $n-1$ and the $n$-body correction to the total electron density. Similarly, the calculation of $T_{s}^{\mathrm{nadd},(n)}$ and $E_{\mathrm{xc}}^{\mathrm{nadd},(n)}$ only requires the electron densities from the subsystem calculations. Further details of our implementation are given in the Computational Details. As they only require the knowledge of electron densities, all contributions to $E_{\text {corr }}^{(n)}$ are accessible both in DFT and in wavefunction-based 

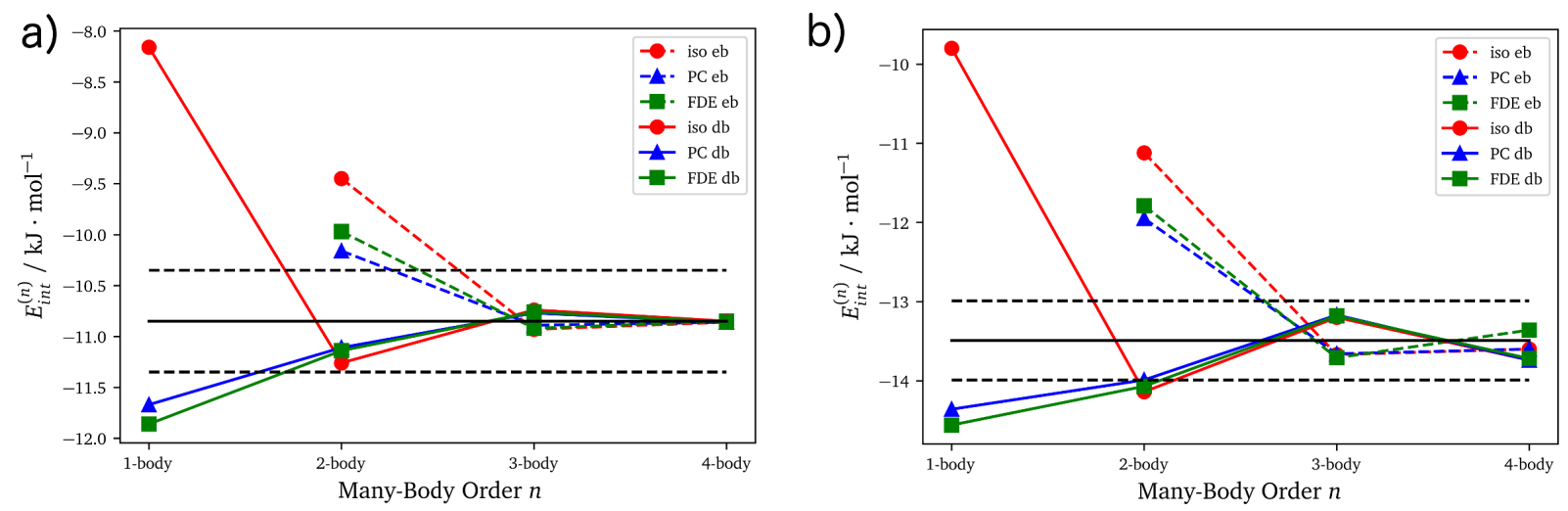

Figure 4: Convergence of the density-based many-body expansion for the interaction energies per molecular fragment, $E_{\text {int,db-MBE }}^{(n)}(\mathrm{in} \mathrm{kJ} / \mathrm{mol})$ of $(\mathrm{a})\left(\mathrm{H}_{2} \mathrm{O}\right)_{5}$ and $(\mathrm{b})\left(\mathrm{H}_{2} \mathrm{O}\right)_{17}$ for DFT/BP86/TZP calculations. Included are results for the db-MBE (solid lines) using isolated subsystems ("iso", red circles) as well as point charge embedding ("PC", blue triangles) as well as frozen-density embedding ("FDE", green squares). The interaction energies obtained with energy-based MBEs (dashed lines, cf. Fig. 2) are included for comparison, The supermolecular reference values are included as solid horizontal line, whereas the dashed horizontal lines indicate a target accuracy of $0.5 \mathrm{~kJ} / \mathrm{mol}$ per fragment. See text for details.

quantum-chemical calculations. Moreover, the all subsystem calculations can either be performed for isolated or for embedded subsystems without affecting the calculation of $E_{\text {corr. }}^{(n)}$. Thus, a db-MBE as introduced here can be consistently combined with any MBE strategy without introducing issues due to possible double counting.

\subsection{Test Case: Water Clusters}

For a first test of the accuracy of density-based MBEs we return to the $\left(\mathrm{H}_{2} \mathrm{O}\right)_{5}$ and $\left(\mathrm{H}_{2} \mathrm{O}\right)_{17}$ water clusters considered above. Table IV lists the interaction energies per molecule obtained with truncated db-MBEs at different expansion orders. These values can be directly compared to those in Table II for the eb-MBEs. The many-body convergence of the energy-based and density-based MBEs is also visualized in Fig. 4.

Whereas in the eb-MBE at least a two-body expansion is required to approximate the 
Table IV: Interaction energies per molecular fragment (in $\mathrm{kJ} / \mathrm{mol}$ ) of the water clusters $\left(\mathrm{H}_{2} \mathrm{O}\right)_{5}$ and $\left(\mathrm{H}_{2} \mathrm{O}\right)_{17}$ obtained from density-based many-body expansions compared to the supermolecular reference value for DFT/BP86/TZP calculations. Included are the isolated db-MBE ("iso") as well as embedded db-MBEs using point charge embedding ("PC") and frozen-density embedding ("FDE"). See text for details.

\begin{tabular}{|c|c|c|c|c|c|c|c|}
\hline & \multirow[b]{2}{*}{ order } & \multicolumn{2}{|l|}{ iso } & \multicolumn{2}{|l|}{$\mathrm{PC}$} & \multicolumn{2}{|c|}{ FDE } \\
\hline & & $E_{\text {int,db-MBE }}^{(n)}$ & $\Delta^{(n)}$ & $E_{\text {int,db-MBE }}^{(n)}$ & $\Delta^{(n)}$ & $E_{\mathrm{int}, \mathrm{db}-\mathrm{MBE}}^{(n)}$ & $\Delta^{(n)}$ \\
\hline \multirow[t]{5}{*}{$\left(\mathrm{H}_{2} \mathrm{O}\right)_{5}$} & 1-body & -8.16 & +2.69 & -11.67 & -0.82 & -11.86 & -1.01 \\
\hline & 2-body & -11.26 & -0.41 & -11.11 & -0.26 & -11.14 & -0.29 \\
\hline & 3-body & -10.74 & +0.09 & -10.77 & +0.08 & -10.76 & +0.09 \\
\hline & 4-body & -10.85 & 0.00 & -10.86 & -0.01 & -10.86 & -0.01 \\
\hline & supermol. & -10.85 & & -10.85 & & -10.85 & \\
\hline \multirow[t]{5}{*}{$\left(\mathrm{H}_{2} \mathrm{O}\right)_{17}$} & 1-body & -9.80 & +3.69 & -14.36 & -0.87 & -14.56 & -1.07 \\
\hline & 2-body & -14.14 & -0.65 & -13.99 & -0.50 & -14.07 & -0.58 \\
\hline & 3-body & -13.20 & +0.29 & -13.17 & +0.32 & -13.18 & +0.31 \\
\hline & 4-body & -13.74 & -0.25 & -13.74 & -0.25 & -13.72 & -0.23 \\
\hline & supermol. & -13.49 & & -13.49 & & -13.49 & \\
\hline
\end{tabular}


interaction energy in a molecular cluster, the db-MBE already provides a reasonable estimate at first order, i.e., for the quantum-chemical calculation performed for the monomers. For the isolated db-MBE, the energy difference to the full supermolecular calculation amounts to $2.69 \mathrm{~kJ} / \mathrm{mol}$ per fragment for $\left(\mathrm{H}_{2} \mathrm{O}\right)_{5}$ and to $3.69 \mathrm{~kJ} / \mathrm{mol}$ per fragment $\left(\mathrm{H}_{2} \mathrm{O}\right)_{17}$. However, for the embedded db-MBEs the error of the one-body db-MBE is below $1.1 \mathrm{~kJ} / \mathrm{mol}$ per fragment for both test cases. This error is comparable to or even smaller than the one of the two-body eb-MBE.

For $\left(\mathrm{H}_{2} \mathrm{O}\right)_{5}$ the error in the interaction energy amounts to $0.41 \mathrm{~kJ} / \mathrm{mol}$ per fragment for the isolated db-MBE and to less than $0.3 \mathrm{~kJ} / \mathrm{mol}$ per fragment for the embedded dbMBEs. For the db-MBE this is only a third of the truncation error of the eb-MBE, while for the embedded db-MBEs the error is reduced to less than half. For the 3-body db-MBE the remaining error in the interaction energy is less than $0.1 \mathrm{~kJ} / \mathrm{mol}$ per fragment, which is similar to the truncation error of the corresponding 3-body eb-MBEs.

For $\left(\mathrm{H}_{2} \mathrm{O}\right)_{17}$ the two-body db-MBE predicts the interaction energy within $0.65 \mathrm{~kJ} / \mathrm{mol}$ for both the isolated and the embedded case. Compared to the two-body eb-MBE, this corresponds to a reduction to almost a fourth in for the isolated MBE and to approximately a third in the embedded MBEs. For the 3-body db-MBE, the error amounts to ca. $0.3 \mathrm{~kJ} / \mathrm{mol}$ per fragment in all cases, which is comparable to the truncation error of the corresponding 3-body eb-MBEs. It is interesting to note that when comparing the results for $\left(\mathrm{H}_{2} \mathrm{O}\right)_{5}$ and $\left(\mathrm{H}_{2} \mathrm{O}\right)_{17}$, in the eb-MBEs the truncation error per fragment seems to increase roughly linearly with the number of fragments. Contrarily, for the one-body db-MBE the errors per fragment are similar for both cluster sizes, and for the two-body db-MBE only a slight increase of the error per fragment is observed for the larger cluster.

The visualization of the above data in Fig. 4 demonstrates that to obtain interaction energies of similar (or even better) accuracy, the db-MBE accelerates the convergence of the MBE by one order. While in the eb-MBE, a 3-body expansion is required to reach 
a)

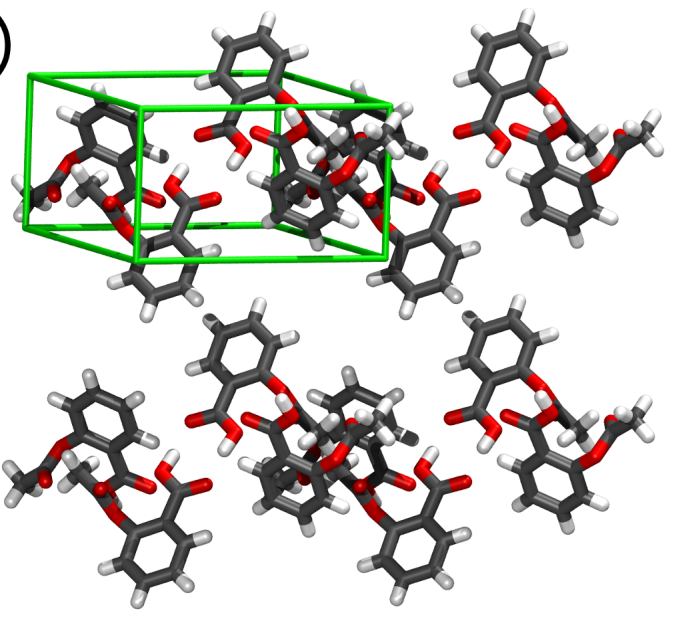

b)

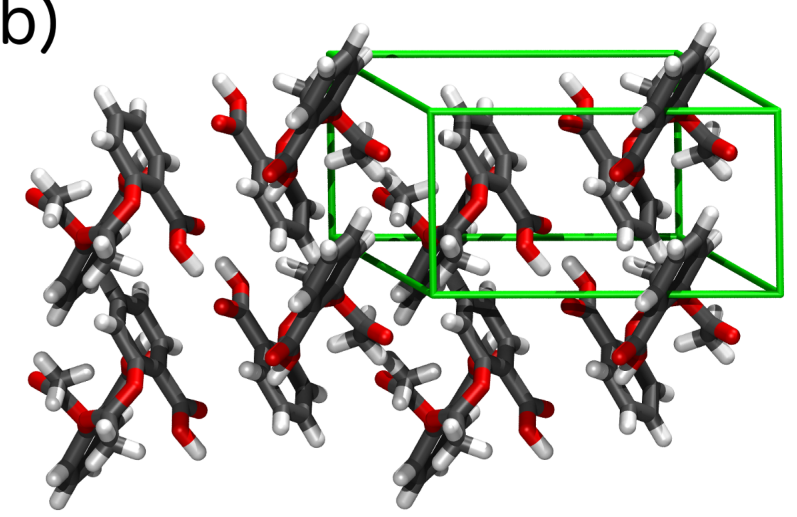

Figure 5: Structures of clusters of 16 aspirin molecules extracted from the crystal structures of (a) form I and (b) form II. The unit cell is shown in green, and the considered clusters correspond to $2 \times 2$ supercells.

a target accuracy of $0.5 \mathrm{~kJ} / \mathrm{mol}$ per fragment, in the db-MBE a two-body expansion is already sufficient. In the case of $\left(\mathrm{H}_{2} \mathrm{O}\right)_{17}$, this corresponds to saving 680 quantumchemical calculations for trimers, which altogether require a substantial computational effort.

\subsection{Test Case: Aspirin Clusters}

As another test case, we consider molecular clusters of 2-acetoxybenzoic acid (aspirin). Aspirin crystallizes in two different polymorphs, dubbed form I and form II [92]. These are almost isoenergetic, which make the computational prediction of the energy differences between the two polymorphs a challenging test case [93]. Here, we consider two clusters of 16 aspirin molecules constructed as $2 \times 2$ supercells of the crystals structures of form I and form II (see Fig. 5). The crystal structures have been obtained from the Cambridge Crystallographic Data Centre (CCDC) with reference codes ACSALA14 (form I) and ACSALA15 (form II).

While in the water clusters, the intermolecular interactions are dominated by hydro- 
Table V: Interaction energies (in $\mathrm{kJ} / \mathrm{mol}$ ) for the clusters of 16 aspirin molecules cut from the crystal structures of form I and form II obtained from energy-based as well as density-based many-body expansions compared to the supermolecular reference value for DFT/BP86/TZP calculations. Included are the isolated MBE ("iso") as well as the frozen-density embedded MBE. See text for details.

\begin{tabular}{|c|c|c|c|c|c|c|c|c|c|}
\hline & \multirow[b]{3}{*}{ order } & \multicolumn{4}{|c|}{ energy-based } & \multicolumn{4}{|c|}{ density-based } \\
\hline & & \multicolumn{2}{|c|}{ iso } & \multicolumn{2}{|c|}{ FDE } & \multicolumn{2}{|c|}{ iso } & \multicolumn{2}{|c|}{ FDE } \\
\hline & & $E_{\text {int }}^{(n)}$ & $\Delta^{(n)}$ & $E_{\text {int }}^{(n)}$ & $\Delta^{(n)}$ & $E_{\text {int }}^{(n)}$ & $\Delta^{(n)}$ & $E_{\text {int }}^{(n)}$ & $\Delta^{(n)}$ \\
\hline \multirow[t]{3}{*}{ form I } & 1-body & & & & & -136.9 & +72.8 & -272.4 & -62.7 \\
\hline & 2-body & -201.9 & +7.9 & -203.4 & +6.4 & -210.7 & -1.0 & -213.2 & -3.5 \\
\hline & supermol. & -209.8 & & -209.8 & & -209.8 & & -209.8 & \\
\hline \multirow[t]{3}{*}{ form II } & 1-body & & & & & -58.7 & -12.8 & -172.6 & -126.7 \\
\hline & 2-body & -33.1 & +12.7 & -31.0 & +14.8 & -47.9 & -2.1 & -51.5 & -5.7 \\
\hline & supermol. & -45.8 & & -45.8 & & -45.8 & & -45.8 & \\
\hline
\end{tabular}

gen bonding, in these aspirin clusters different types of intermolecular interactions, including hydrogen bonding, $\pi-\pi$ stacking, dipole-dipole interaction, dipole $-\pi$ interactions, and dispersion interactions, are competing. Note that while our aspirin form I cluster contains four hydrogen-bonded dimers, our form II cluster only contains two hydrogenbonded dimers. Here, we compare the interaction energies obtained with the different MBEs to the supermolecular reference calculation. All calculations are performed using DFT/BP86/TZP. We note that no dispersion correction is included in our calculations, but this does not affect the comparison of the truncated MBE and the supermolecular results.

The interaction energies calculated for the form I and form II aspirin clusters obtained with the isolated and frozen-density embedded eb-MBEs as well as db-MBEs up to twobody contributions are given in Table $\mathrm{V}$. These are compared to the interaction energies from the corresponding supermolecular calculations. Because only clusters of the same size are compared, we now show the total interaction energies instead of the interaction energies per monomer discussed for the water clusters. When using calculations for isolated 
subsystems, the eb-MBE truncated after two-body contributions results in a truncation error of $7.9 \mathrm{~kJ} / \mathrm{mol}$ for form I and of $12.8 \mathrm{~kJ} / \mathrm{mol}$ for form II. Using FDE in the subsystem calculations slightly improves the interaction energy for form I, but worsens the result for form II.

With the db-MBE, the one-body expansion (i.e., using only the calculations for monomers) provides a first estimate of the interaction energies, but with sizable errors between +72.8 and $-126.7 \mathrm{~kJ} / \mathrm{mol}$ both for the isolated and the frozen-density embedded case. These can be attributed to the shortcoming of the employed nonadditive kinetic-energy functional for weak intermolecular interactions $[94,95]$. When including two-body terms in the isolated db-MBE, the errors in the interaction energies are reduced to $0.6 \mathrm{~kJ} / \mathrm{mol}$ for form I and $2.3 \mathrm{~kJ} / \mathrm{mol}$ for form II, i.e., the accuracy improves by more than a factor of five compared to the isolated eb-MBE. Altogether, the error of the two-body iso-db-MBE is below chemical accuracy for both aspirin clusters. For the frozen-density embedded db-MBE, the errors in the interaction energies of $3.5 \mathrm{~kJ} / \mathrm{mol}$ for form I and $5.7 \mathrm{~kJ} / \mathrm{mol}$ are again significantly smaller than for the eb-MBE by approximately a factor of two. However, these are somewhat larger than for the iso-db-MBE. We attribute this to the lack of a self-consistent optimization of the subsystem electron densities.

\section{Conclusions}

We have explored the combination of MBEs with quantum embedding methods. The MBE constitutes a fragmentation method for the quantum-chemical treatment of large molecular clusters and crystals that systematically converges towards the exact supermolecular result. However, it only becomes formally exact if all $n$-body contributions up to the number of molecular fragments are included. A truncation at low order has to be applied to gain a computational advantage compared to a full, supermolecular cal- 
culation. However, this truncation inevitably introduces an error. On the other hand, quantum-embedding methods and related quantum-chemical subsystem methods are in principle able to provide the exact supermolecular result from the fragment calculations (i.e., including only one-body terms). However, to be computationally feasible all such methods introduce approximations of some sort, and their accuracy might be difficult to predict.

In this work, we have combined these two complementary approaches, i.e., the systematically improvable truncated MBE with the formally exact subsystem DFT and frozendensity embedding (FDE) theory. First, we have applied FDE for including the effect of the environment into the subsystem calculations necessary in the FDE. We find that for water clusters, this can reduce the errors in the interaction energy for an eb-MBE truncated at low order compared to the use of calculations for the isolated subsystems. However, using FDE hardly improves upon classical point charge embedding. Furthermore, for the test case of aspirin clusters no benefits of frozen-density embedded subsystem calculations are found compared to the isolated eb-MBE.

However, we find that the use of a suitable embedding potential in the subsystem calculations significantly improves the convergence of a MBE for the electron density. In fact, with the exact FDE embedding potential, already a one-body expansion (i.e., the sum of fragment densities) would reproduce the correct supermolecular density. With an approximate embedding potential, we find that the embedded MBE converges rapidly for the electron density, and already a two-body expansion is often sufficiently accurate.

A further analysis shows that even if the MBE of the electron density is converged, the conventional eb-MBE will not yield the exact supermolecular energy. At first order, the eb-MBE misses the interaction between the monomer electron densities, while at second order it misses the interaction of the dimer corrections, $\Delta \rho_{I J}$, with the electron densities of the other subsystems. This can be alleviated with the db-MBE introduced in 
the present work. By inserting the many-body expanded electron density into the total energy functional, it becomes possible to exploit the rapid convergence of the MBE for the electron density to improve the energies obtained from the eb-MBE.

We find that the use of a db-MBE can accelerate the many-body convergence by one order compared to the eb-MBE. For our test cases, accurate interaction energies can be obtained from a db-MBE including only one- and two-body terms. For the considered water clusters, the errors in the interaction energies are below $1 \mathrm{~kJ} / \mathrm{mol}$ per fragment, whereas they are smaller than $4 \mathrm{~kJ} / \mathrm{mol}$ for the considered clusters of 16 aspirin molecules. With an eb-MBE, the inclusion of 3-body terms is mandatory to achieve a such an accuracy. As the benchmarks presented here are limited, we plan to present more comprehensive tests of the accuracy of db-MBEs elsewhere.

Conceptually, there are two different perspectives on the db-MBE. First, it can be viewed as an ONIOM-style composite method [56-58], in which a truncated MBE using a suitable quantum-chemical method is used as high-level method while an orbital-free DFT treatment using an approximate kinetic-energy functional is used as low-level method (cf. Ref. [96]). Thus, compared to other composite schemes based on a truncated MBE [59-62], the use of, e.g., a polarizable force field for approximating the neglected manybody contributions, is replaced by an interaction energy correction that depends on the subsystems' electron densities. Second, the db-MBE can be viewed as an extension of subsystem-DFT [12] that corrects for insufficiencies of approximate nonadditive kineticenergy functional by pairwise (and possibly higher-order) corrections.

While here we only employed KS-DFT for the subsystem calculations within the dbMBE, the presented methodology can be combined with any quantum-chemical method, including high-level wave-function based method. As the theory is formulated only in terms of the subsystem electron densities, it is particularly easy to combine with different quantum-chemical methods and MBE strategies, without introducing problems due to a 
potential double counting of energy contributions. In combination with periodic densitybased embedding schemes $[28,97]$, the db-MBE thus provides a intriguing approach for the accurate quantum-chemical treatment of molecular crystals. In such applications, the use of accurate nonlocal kinetic-energy functionals recently developed by Pavanello and coworkers $[98,99]$ might be particularly promising.

\section{Computational Details}

All quantum-chemical calculations have been performed using DFT as implemented in the Amsterdam Density-Functional (ADF) program package $[100,101]$. The BP86 xcfunctional $[83,84]$ and a triple-zeta plus polarization (TZP) basis set of Slater-type orbitals [85] have been used throughout. All subsystem energies have been obtained with ADF's total energy implementation [94]. To be sure to avoid numerical precision issues [37], we used a Becke integration grid of "very good" accuracy [102]. For the frozendensity embedded MBE calculations we employed ADF's implementation of FDE [103]. To ensure all energy terms are obtained with the required accuracy, the full supermolecular integration grid is used in all FDE calculations as well as for the evaluation of the interaction energy terms in the db-MBE. The PW91k nonadditive kinetic energy functional [104] has been used were applicable.

To automatize the subsystem calculations needed to perform the eb-MBE, we utilized the PyADF scripting framework [105]. We have implemented the additional interaction energy terms needed for the db-MBE in PyADF, making use of our recently developed PyEmbed module, which provides a stand-alone implementation of the subsystem DFT embedding potential and interaction energy terms. The $n$-body nonadditive xc and kinetic energy [see Eqs. (32) and (33)] have been evaluated using the XCFun library [106, 107]. The electrostatic interaction energies are calculated in PyEmbed by numerical integration 
from the electron densities and the nuclear and Coulomb potentials exported from ADF according to Eqs. (28), (34), and (35). Here, the necessary fit corrections are included as described in Refs. [94, 108].

We note that by using subsystem-specific grids instead of relying on the supermolecular integration grid in all steps, the performance of our current pilot implementation can be improved. In our future work, we will explore these possibilities in order to provide an efficient implementation of density-based many-body expansions.

Cartesian coordinates of all molecular clusters used as test cases in this work are available at https://doi.org/10.26434/chemrxiv.11816739.

\section{Acknowledgements}

We are indebted to Dr. André Severo Pereira Gomes (Laboratoire PhLAM, CNRS UMR 8523 and Université de Lille) for contributions to the development of PyEmbed. We further thank Roland Pollack (TU Braunschweig) for performing initial calculations at an early stage of this project.

\section{Author Contributions}

CRJ designed the study and supervised the project. DSM and CRJ developed the methodology and implemented the software. DSM performed all calculations, analyzed the data, and wrote a first draft of the manuscript. CRJ wrote the final version of the manuscript, which both authors critically revised and approved. 


\section{References}

[1] M. S. Gordon, D. G. Fedorov, S. R. Pruitt, and L. V. Slipchenko, Chem. Rev. 112, $632(2012)$.

[2] S. R. Pruitt, C. Bertoni, K. R. Brorsen, and M. S. Gordon, Acc. Chem. Res. 47, 2786 (2014).

[3] K. Raghavachari and A. Saha, Chem. Rev. 115, 5643 (2015).

[4] L. O. Jones, M. A. Mosquera, G. C. Schatz, and M. A. Ratner, J. Am. Chem. Soc. 142, $3281(2020)$.

[5] A. S. P. Gomes and Ch. R. Jacob, Annu. Rep. Prog. Chem., Sect. C 108, 222 (2012).

[6] A. Goez and J. Neugebauer, Embedding Methods in Quantum Chemistry, in Frontiers of Quantum Chemistry, edited by M. J. Wójcik, H. Nakatsuji, B. Kirtman, and Y. Ozaki, pages 139-179, Springer, Singapore, 2018, DOI: 10.1007/978-981-10$5651-2 \_7$.

[7] B. Mennucci, WIREs Comput. Mol. Sci. 2, 386 (2012).

[8] F. Lipparini and B. Mennucci, J. Chem. Phys. 144, 160901 (2016).

[9] H. M. Senn and W. Thiel, Angew. Chem. Int. Ed. 48, 1198 (2009).

[10] S. Ahmadi, L. B. Herrera, M. Chehelamirani, J. Hostaš, S. Jalife, and D. R. Salahub, Int. J. Quantum Chem. 118, e25558 (2018).

[11] U. N. Morzan, D. J. Alonso de Armiño, N. O. Foglia, F. Ramírez, M. C. González Lebrero, D. A. Scherlis, and D. A. Estrin, Chem. Rev. 118, 4071 (2018).

[12] Ch. R. Jacob and J. Neugebauer, WIREs Comput. Mol. Sci. 4, 325 (2014). 
[13] F. Libisch, C. Huang, and E. A. Carter, Acc. Chem. Res. 47, 2768 (2014).

[14] T. A. Wesolowski, S. Shedge, and X. Zhou, Chem. Rev. 115, 5891 (2015).

[15] Q. Sun and G. K.-L. Chan, Acc. Chem. Res. 49, 2705 (2016).

[16] S. J. R. Lee, M. Welborn, F. R. Manby, and T. F. Miller, Acc. Chem. Res. 52, 1359 (2019).

[17] J. Neugebauer, Ch. R. Jacob, T. A. Wesolowski, and E. J. Baerends, J. Phys. Chem. A 109, 7805 (2005).

[18] R. E. Bulo, Ch. R. Jacob, and L. Visscher, J. Phys. Chem. A 112, 2640 (2008).

[19] T. D. Crawford, A. Kumar, K. P. Hannon, S. Höfener, and L. Visscher, J. Chem. Theory Comput. 11, 5305 (2015).

[20] C. König and J. Neugebauer, Phys. Chem. Chem. Phys. 13, 10475 (2011).

[21] J. Neugebauer, J. Veldstra, and F. Buda, J. Phys. Chem. B 115, 3216 (2011).

[22] C. König and J. Neugebauer, J. Chem. Theory Comput. 9, 1808 (2013).

[23] S. J. Bennie, M. W. van der Kamp, R. C. R. Pennifold, M. Stella, F. R. Manby, and A. J. Mulholland, J. Chem. Theory Comput. 12, 2689 (2016).

[24] X. Zhang, S. J. Bennie, M. W. van der Kamp, D. R. Glowacki, F. R. Manby, and A. J. Mulholland, R. Soc. Open Sci 5, 171390 (2018).

[25] H. Jiang, M. Kammler, F. Ding, Y. Dorenkamp, F. R. Manby, A. M. Wodtke, T. F. Miller, A. Kandratsenka, and O. Bünermann, Science 364, 379 (2019).

[26] P. Cortona, Phys. Rev. B 46, 2008 (1992).

[27] M. Iannuzzi, B. Kirchner, and J. Hutter, Chem. Phys. Lett. 421, 16 (2006). 
[28] A. Genova, D. Ceresoli, and M. Pavanello, J. Chem. Phys. 141, 174101 (2014).

[29] M. Welborn, T. Tsuchimochi, and T. Van Voorhis, J. Chem. Phys. 145, 074102 (2016).

[30] G. J. O. Beran, Chem. Rev. 116, 5567-5613 (2016).

[31] S. Wen and G. J. O. Beran, J. Chem. Theory Comput. 8, 2698 (2012).

[32] J. Yang, W. Hu, D. Usvyat, D. Matthews, M. Schütz, and G. K.-L. Chan, Science 345, 640 (2014).

[33] H. Stoll and H. Preuß, Theor. Chim. Acta 46, 11 (1977).

[34] I. G. Kaplan, Theory of molecular interactions, Elsevier, Amsterdam, 1986.

[35] K. Rościszewski, B. Paulus, P. Fulde, and H. Stoll, Phys. Rev. B 60, 7905 (1999).

[36] J. Cui, H. Liu, and K. D. Jordan, J. Phys. Chem. B 110, 18872 (2006).

[37] R. M. Richard, K. U. Lao, and J. M. Herbert, J. Chem. Phys. 141, 014108 (2014).

[38] K.-Y. Liu and J. M. Herbert, J. Chem. Phys. 147, 161729 (2017).

[39] P. Jerabek, O. R. Smits, J.-M. Mewes, K. A. Peterson, and P. Schwerdtfeger, J. Phys. Chem. A 123, 4201 (2019).

[40] J. M. Herbert, J. Chem. Phys. 151, 170901 (2019).

[41] I. G. Kaplan, R. Santamaria, and O. Novaro, Mol. Phys. 84, 105 (1995).

[42] C. König and O. Christiansen, J. Chem. Phys. 145, 064105 (2016).

[43] D. Madsen, O. Christiansen, and C. König, Phys. Chem. Chem. Phys. 20, 3445 (2018).

[44] E. E. Dahlke and D. G. Truhlar, J. Chem. Theory Comput. 3, 46 (2006). 
[45] E. E. Dahlke and D. G. Truhlar, J. Chem. Theory Comput. 4, 1 (2008).

[46] P. J. Bygrave, N. L. Allan, and F. R. Manby, J. Chem. Phys. 137, 164102 (2012).

[47] S. Wen and G. J. O. Beran, J. Chem. Theory Comput. 7, 3733 (2011).

[48] Ch. R. Jacob, J. Neugebauer, L. Jensen, and L. Visscher, Phys. Chem. Chem. Phys. 8, 2349 (2006).

[49] R. Kevorkyants, X. Wang, D. M. Close, and M. Pavanello, J. Phys. Chem. B 117, 13967 (2013).

[50] J. Gao and Y. Wang, J. Chem. Phys. 136, 071101 (2012).

[51] K. Kitaura, E. Ikeo, T. Asada, T. Nakano, and M. Uebayasi, Chem. Phys. Lett. 313, 701 (1999).

[52] D. G. Fedorov and K. Kitaura, J. Phys. Chem. A 111, 6904 (2007).

[53] D. G. Fedorov, N. Asada, I. Nakanishi, and K. Kitaura, Acc. Chem. Res. 47, 2846 (2014).

[54] S. P. Veccham, J. Lee, and M. Head-Gordon, J. Chem. Phys. 151, 194101 (2019).

[55] M. E. Fornace, J. Lee, K. Miyamoto, F. R. Manby, and T. F. Miller, J. Chem. Theory Comput. 11, 568 (2015).

[56] M. Svensson, S. Humbel, R. D. J. Froese, T. Matsubara, S. Sieber, and K. Morokuma, J. Phys. Chem. 100, 19357 (1996).

[57] L. W. Chung, H. Hirao, X. Li, and K. Morokuma, WIREs Comput. Mol. Sci. 2, $327(2012)$.

[58] L. W. Chung et al., Chem. Rev. (2015).

[59] G. S. Tschumper, Chem. Phys. Lett. 427, 185 (2006). 
[60] N. J. Mayhall and K. Raghavachari, J. Chem. Theory Comput. 7, 1336 (2011).

[61] G. J. O. Beran, J. Chem. Phys. 130, 164115 (2009).

[62] G. J. O. Beran and K. Nanda, J. Phys. Chem. Lett. 1, 3480 (2010).

[63] T. A. Wesolowski and A. Warshel, J. Phys. Chem. 97, 8050 (1993).

[64] N. Govind, Y. A. Wang, A. J. R. da Silva, and E. A. Carter, Chem. Phys. Lett. 295, 129 (1998).

[65] A. S. P. Gomes, Ch. R. Jacob, and L. Visscher, Phys. Chem. Chem. Phys. 10, 5353 (2008).

[66] A. S. P. Gomes, Ch. R. Jacob, F. Réal, L. Visscher, and V. Vallet, Phys. Chem. Chem. Phys. 15, 15153 (2013).

[67] R. G. Parr and W. Yang, Density-Functional Theory of Atoms and Molecules, Oxford University Press, Oxford, 1989.

[68] E. Engel and R. M. Dreizler, Density Functional Theory: An Advanced Course, Springer, Heidelberg, 2011.

[69] W. Li, S. Li, and Y. Jiang, J. Phys. Chem. A 111, 2193 (2007).

[70] R. M. Richard and J. M. Herbert, J. Chem. Phys. 137, 064113 (2012).

[71] R. M. Richard, K. U. Lao, and J. M. Herbert, Acc. Chem. Res. 47, 2828 (2014).

[72] K. U. Lao, K.-Y. Liu, R. M. Richard, and J. M. Herbert, J. Chem. Phys. 144, 164105 (2016).

[73] S. Fux, Ch. R. Jacob, J. Neugebauer, L. Visscher, and M. Reiher, J. Chem. Phys. 132, $164101(2010)$. 
[74] J. P. Unsleber, J. Neugebauer, and Ch. R. Jacob, Phys. Chem. Chem. Phys. 18, 21001 (2016).

[75] T. A. Wesolowski, H. Chermette, and J. Weber, J. Chem. Phys. 105, 9182 (1996).

[76] T. A. Wesolowski, J. Chem. Phys. 106, 8516 (1997).

[77] Y. Chen and H. Li, J. Phys. Chem. A 114, 11719 (2010).

[78] G. A. Cisneros, K. T. Wikfeldt, L. Ojamäe, J. Lu, Y. Xu, H. Torabifard, A. P. Bartók, G. Csányi, V. Molinero, and F. Paesani, Chem. Rev. 116, 7501 (2016).

[79] C. H. Pham, S. K. Reddy, K. Chen, C. Knight, and F. Paesani, J. Chem. Theory Comput. 13, 1778 (2017).

[80] K.-Y. Liu and J. M. Herbert, J. Chem. Theory Comput. 16, 475 (2020).

[81] J. D. Bernal and R. H. Fowler, J. Chem. Phys. 1, 515 (1933).

[82] American Mineralogist Crystal Structure Database, ID 0017930, URL: http://rruff.geo.arizona.edu/AMS/amcsd.php.

[83] A. D. Becke, Phys. Rev. A 38, 3098 (1988).

[84] J. P. Perdew, Phys. Rev. B 33, 8822 (1986).

[85] E. Van Lenthe and E. J. Baerends, J. Comput. Chem. 24, 1142 (2003).

[86] W. L. Jorgensen, J. Chandrasekhar, J. D. Madura, R. W. Impey, and M. L. Klein, J. Chem. Phys. 79, 926 (1983).

[87] T. A. Wesolowski and J. Weber, Chem. Phys. Lett. 248, 71 (1996).

[88] H. R. Leverentz and D. G. Truhlar, J. Chem. Theory Comput. 5, 1573 (2009), Publisher: American Chemical Society, DOI: 10.1021/ct900095d. 
[89] J. Harris, Phys. Rev. B 31, 1770 (1985).

[90] F. W. Averill and G. S. Painter, Phys. Rev. B 41, 10344 (1990).

[91] P. Cortona, Phys. Rev. B 44, 8454 (1991).

[92] A. D. Bond, R. Boese, and G. R. Desiraju, Angew. Chem. Int. Ed. 46, 618 (2007).

[93] S. Wen and G. J. O. Beran, Cryst. Growth Des. 12, 2169 (2012).

[94] A. W. Götz, S. M. Beyhan, and L. Visscher, J. Chem. Theory Comput. 5, 3161 (2009).

[95] D. Schlüns, K. Klahr, C. Mück-Lichtenfeld, L. Visscher, and J. Neugebauer, Phys. Chem. Chem. Phys. 17, 14323 (2015).

[96] T. Klüner, N. Govind, Y. A. Wang, and E. A. Carter, J. Chem. Phys. 116, 42 (2002).

[97] J. Tölle, A. Severo Pereira Gomes, P. Ramos, and M. Pavanello, Int. J. Quantum Chem. 119, e25801 (2019).

[98] W. Mi, A. Genova, and M. Pavanello, J. Chem. Phys. 148, 184107 (2018).

[99] W. Mi and M. Pavanello, J. Phys. Chem. Lett. (2019).

[100] G. te Velde, F. M. Bickelhaupt, E. J. Baerends, C. Fonseca Guerra, S. J. A. van Gisbergen, J. G. Snijders, and T. Ziegler, J. Comput. Chem. 22, 931 (2001).

[101] Theoretical Chemistry, Vrije Universiteit Amsterdam, AdF, Amsterdam density functional program, URL: http://www.scm.com.

[102] M. Franchini, P. H. T. Philipsen, and L. Visscher, J. Comput. Chem. 34, 1819 (2013).

[103] Ch. R. Jacob, J. Neugebauer, and L. Visscher, J. Comput. Chem. 29, 1011 (2008). 
[104] A. Lembarki and H. Chermette, Phys. Rev. A 50, 5328 (1994).

[105] Ch. R. Jacob, S. M. Beyhan, R. E. Bulo, A. S. P. Gomes, A. W. Götz, K. Kiewisch, J. Sikkema, and L. Visscher, J. Comput. Chem. 32, 2328 (2011).

[106] U. Ekström, L. Visscher, R. Bast, A. J. Thorvaldsen, and K. Ruud, J. Chem. Theory Comput. 6, 1971 (2010).

[107] U. Ekström et al., XCFun: Exchange-Correlation functionals with arbitrary order derivatives, 2019, URL: https://github.com/dftlibs/xcfun.

[108] D. Schlüns, M. Franchini, A. W. Götz, J. Neugebauer, Ch. R. Jacob, and L. Visscher, J. Comput. Chem. 38, 238 (2017). 